\title{
Development of Age Determination Technique Part 2: Image Processing for Age Determination Based on the Annuli of a Chum Salmon Scale
}

\author{
Masanobu Yoshida ${ }^{1}$, Hiroyuki Shioya ${ }^{1}$, Yasuyuki Miyakoshi ${ }^{2}$, Fumi Yamaguch $^{3}$, and Hirokazu Urabe ${ }^{3}$ \\ ${ }^{1}$ Muroran Institute of Technology, 27-1 Mizumoto, Murorani, Hokkaido, Japan \\ ${ }^{2}$ Kitami Salmon Enhancement Program Association, 5-17 Shinmachi 1, Abashiri, Hokkaido, Japan \\ ${ }^{3}$ Hokkaido Research Organization, Fisheries Department, Salmon and Freshwater Fisheries Research Institute, 3- \\ 373 Kitakashiwagi, Eniwa, Hokkaido, Japan
}

Keywords: salmon scale, age identification, Fourier transform, image processing

\section{Introduction}

In recent years, the poor catch of chum salmon has continued in Japan, and research aimed at monitoring the stock status and elucidating the factors that cause fluctuations in stock has been attracting attention. It takes a lot of time to perform age-assessment work by hand. Information technology has been developed and used for solving various problems in engineering and science fields. Previous work on computerized age-determination has been completed (Endo et al. 1997; Mitani et al. 1988); however, the automation process was not established, and we need to solve the problem associated with huge salmon samples. We constructed new age-assessment technology using advanced image processing to automate the work for scale samples in order to contribute to the investigation of salmon return data in Hokkaido.

\section{Salmon scale data}

The salmon scale has been corrected for the purpose of investigating the age distribution of salmon returns. The data of 20,000 tails of chum salmon are processed every year. The scales are cleaned and transcribed on a plastic plate, known as a replica sample. Three images in Fig. 1 present a chum salmon, the press machine, and a replica sample, respectively. The press machine has been used for making replica samples to produce an engraving print. The objective of using a print-type plate is the preservation of the salmon scale. The curved and striped annuli patterns are arranged around the center of the scale. The narrow annuli indicate a period in each year of slow growth, and the age of the salmon is determined by counting the number of narrow annuli patterns, starting from the center of the scale and moving in a straight line to the edge. The three images of Fig. 2 present the microscope images of scales from the replica samples. The left figure presents the microscope scale image without back noise. The contour of scale is clear, and it is easy to extract the scale from the whole image. The center and left figures present the scale images with the back noise. The noise is ascribable to the distorted plastic plate by the heat press. In the age determination of the salmon scale, the magnifying glass device is often used to extract the scale with the detailed annuli structure. In this case, the back noise of the replica samples does not affect the age determination. Concerning the computational work using the salmon scale, the contrivance to remove such back noise is helpful to obtain clear scale image data.
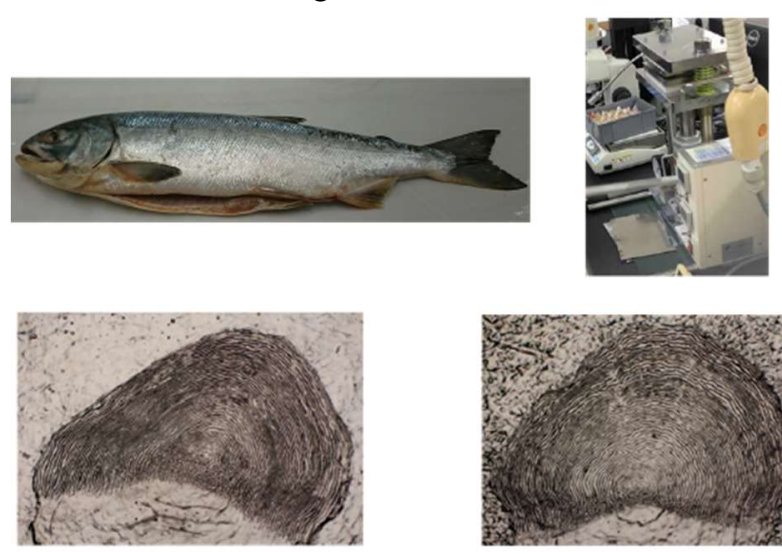

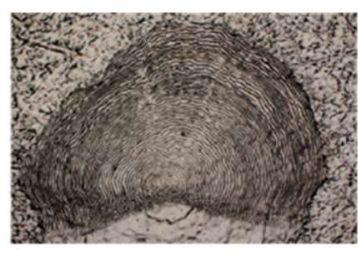

Fig. 1. Chum salmon (left), press machine (middle), and replica sample (right).

Age determination based on image processing

There are two factors important for the age assessment of chum salmon: one is to properly locate the center of the scale, and the other is the ability to find the narrow annuli. It is not easy to find all parts of the annuli on a scale. 
Every year, 20,000 tails of chum salmon are caught for investigating the distribution of salmon returns in Hokkaido prefecture, Japan. Therefore, an automatic system for reading the age data from scales is helpful to shorten the work of the fishery-data research section. The back noise can be removed by a combination of the binary filters. An example of the segmentation is presented in Fig. 3. The shape of the scale is close to a half circle, and a vertical direction of the annuli is effective to find the center. However, the shape of the scale is not a half circle. Therefore, it is possible that a high density partial-region with multi-directional annuli includes the center. We introduced a new iterated procedure to obtain the maximum density region in each step.
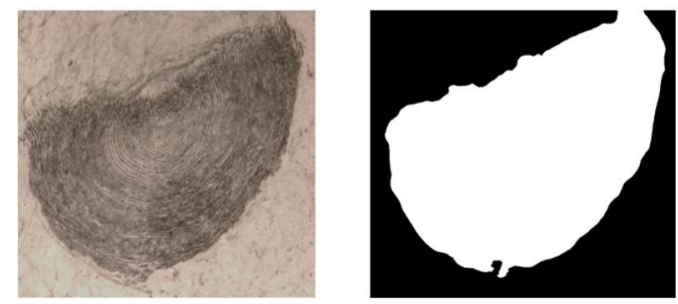

Fig. 3. Original image of salmon scale and binary filter for the segmentation.

Figure 4 presents the new procedure that is used to find the center of a scale. The Fourier transform of a cropped region of the scale image was obtained. The Fourier intensity map indicated the vertical direction of the annuli. Then we obtain the many directions using the Fourier intensities of the scale. It is possible that the region with maximum density of directions is an estimated center. Establishing an accurate center, several iterations of the process with the Fourier transform were introduced. Figure 5 presents the process to have an accurate center of the scale with four-times iterations. By using the extracted center of the scale, the interval frequency of the line through the center indicates the annuli. The total frequency distribution of all lines through the center was presented in the right image in Fig. 6. The left image in Fig. 6 presents the obtained annuli with blue lines. The lines were almost correct as the annuli for the age determination.

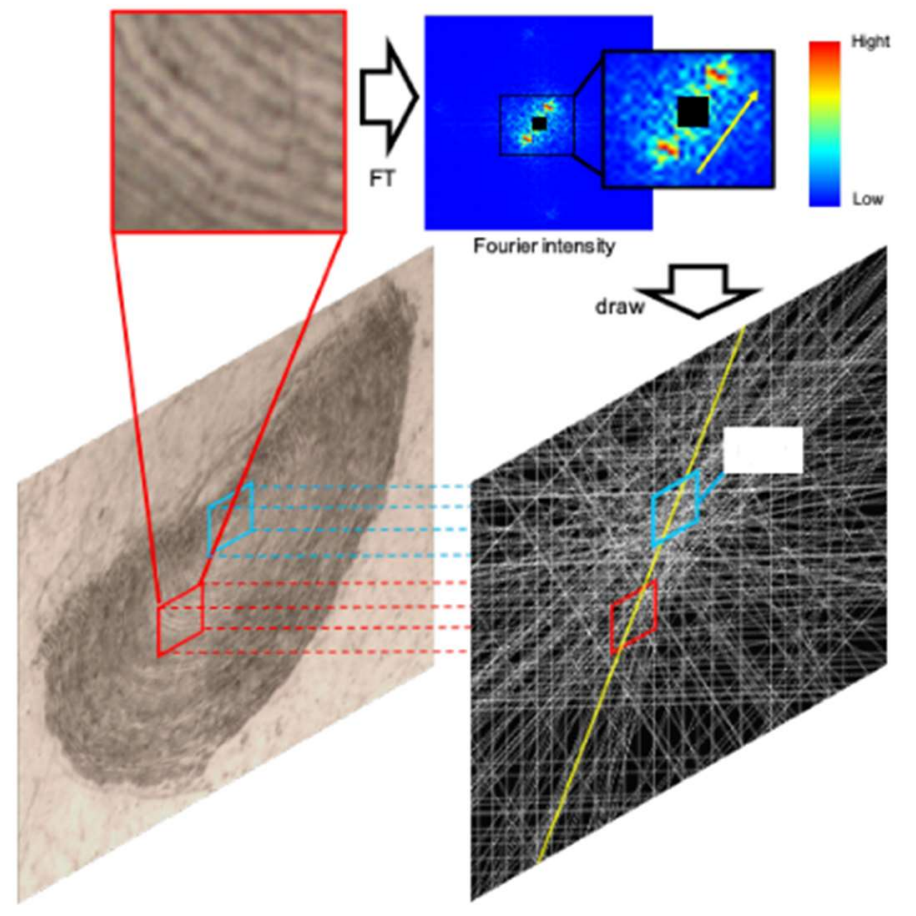

Fig. 4. Center detection of salmon scale using the Fourier transform and maximum density of direction. 

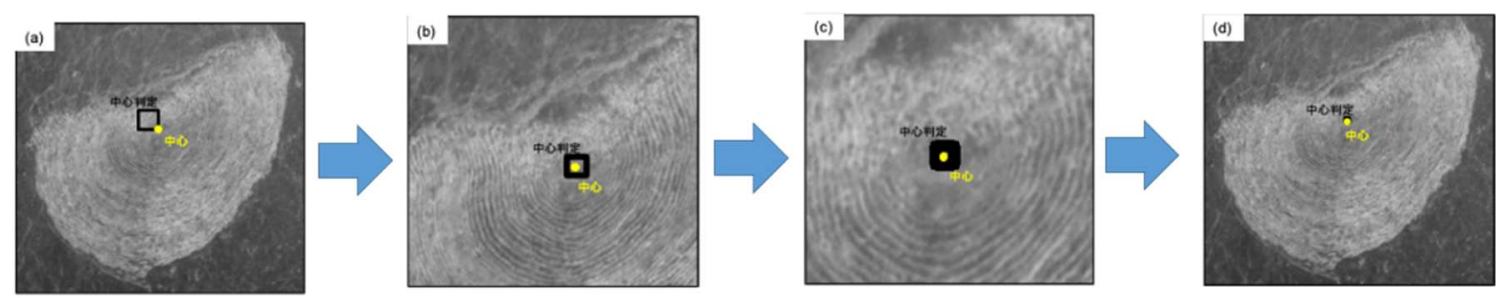

Fig. 5. An accurate center detection process using the iterated Fourier transform and maximum density of directions.
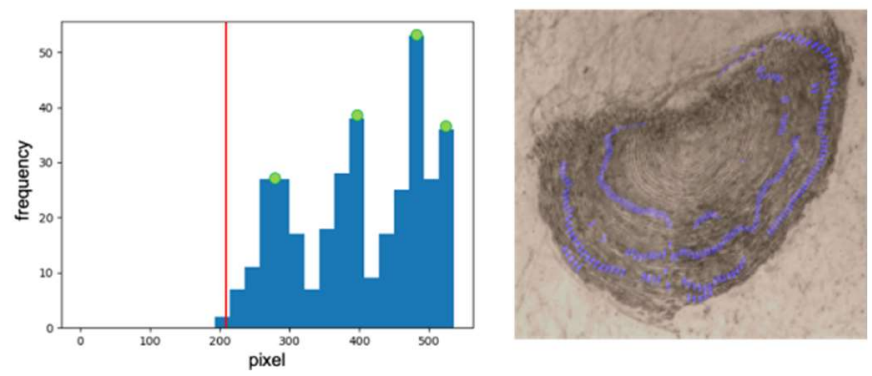

Fig. 6. Total interval frequency using all lines through the extracted center (left). Obtained annuli with the blue lines by using the interval frequency (right).

Summary

In age determination work, replica samples have been used. The samples are good for preserving the scale sample with primitive scale form. However, such plastic plate samples are not adequate to analyze the salmon scale. Therefore, we introduce the fundamental method to have a clear scale image and to obtain the center of the scale with the Fourier transform and maximization of the direction density. It is useful to perform the computational process of a scale analysis system. As a result, the annuli for the age assessment were successfully extracted, and the distribution of the salmon scale leads to the age. The development and refinement of the annuli system will be our future work for investigating the salmon returns in Hokkaido.

\section{REFERENCES}

Endo, Y., O. Watari, and M. Igarashi. 1997. Study on development of age analyzer based on scale patterns. Journal of the Faculty of Marine Science and Technology-Tokai Univ. 44: 1-10. (In Japanese)

Mitani, H., M. Igarashi, and O. Watari. 1988. A study of the age-determination of chum salmon (Oncorhynchus keta) using personal computer for scale reading. Journal of the Faculty of Marine Science and Technology 26: 145-154. (In Japanese) 